\title{
Trends in survival based on treatment modality in patients with pancreatic cancer: a population-based study
}

\author{
S. Shakeel $\mathrm{MPH}^{*}$ C. Finley $\mathrm{MD}^{\dagger}$ G. Akhtar-Danesh MD, ${ }^{\dagger}$ H.Y. Seow $\mathrm{PhD}_{1}^{\ddagger \S}$ and N. Akhtar-Danesh $\mathrm{PhD}^{\ddagger \|}$
}

\begin{abstract}
Background Pancreatic cancer (PCC) is one of the most lethal types of cancer, and surgery remains the optimal treatment modality for patients with resectable tumours. The objective of the present study was to examine and compare trends in the survival rate based on treatment modality in patients with PCC.

Methods This population-based retrospective analysis included all patients with known-stage PCC in Ontario between 2007 and 2015. Flexible parametric models were used to conduct the survival analysis. Survival rates were calculated based on treatment modality, while adjusting for patient- and tumour-specific covariates.

Results The study included 6437 patients. We found no noticeable improvement in survival for patients with stage III or IV tumours; however, for stage I disease, the 1-, 2-, and 5-year survival rates increased over time to $81 \%$ from $51 \%$, to $71 \%$ from $35 \%$, and to $61 \%$ from $22 \%$ respectively. Most improvements were seen for surgical modalities, with 2-year survivals increasing to $89 \%$ from $65 \%$ for distal pancreatectomy (DP) without radiation (RT) or chemotherapy (CTx), to $65 \%$ from $37 \%$ for DP plus RT or CTX, to $60 \%$ from $44 \%$ for Whipple-only, and to $50 \%$ from $36 \%$ for Whipple plus RT or CTx. Lastly, 5 -year survival improved to $81 \%$ from $52 \%$ for DP only, to $41 \%$ from $12 \%$ for DP plus RT or CTx, to $49 \%$ from $25 \%$ for Whipple-only, and to $26 \%$ from $12 \%$ for Whipple plus RT or CTX.
\end{abstract}

Conclusions Most cases of PCC continue to be diagnosed at a late stage, with poor short-term and long-term prognoses. After adjustment for patient age, sex, and year of diagnosis, the survival for stage I tumours and for surgical modalities increased over time. Further research is needed to identify the reasons for improvement in survival during the study period.

Key Words Pancreatic cancer, treatment modalities, survival trends

Curr Oncol. 2020 February;27(1):e1-e8

www.current-oncology.com

\section{INTRODUCTION}

Pancreatic cancer (PCC) is one of the most lethal types of cancer. In Canada, the 5 -year survival rate is $8 \%$, which is one of the lowest rates for all cancers in adults ${ }^{1}$. According to Canadian Cancer Statistics 2017, PCC could soon become the 3 rd leading cause of cancer death in Canada ${ }^{2}$. In the United States, the overall 5-year survival for PCC is also reported to be $8 \%$ (2006-2012), ranging from $3 \%$ to $29 \%$ depending on the disease stage ${ }^{3}$.

Surgery remains the optimal modality for a select group of patients with resectable tumours. However, curative surgical intervention applies to fewer than $20 \%$ of cases, mostly because of late stage at diagnosis or spread to vascular structures around the tumour ${ }^{4-7}$. Treatment modalities and associated survival are dictated by age, comorbidities, cancer stage, location, size, vascular involvement, and extent of tumour spread ${ }^{8,9}$. Improved strategies for earlier detection of tumours, together with neoadjuvant treatment, can increase the number of resectable cases, all of which can improve survival ${ }^{10}$.

However, despite an ability to carry out more complicated surgical procedures, improvements in survival have been limited. Although 1-year survival rates have increased, as seen in studies from Australia, Europe, the United States, and Canada, minimal changes in long-term survival have 
been reported ${ }^{9,11-15}$. One of the challenges in interpreting the prior literature is that analyses have not been adjusted for the concurrent effects of stage and treatments on the associated survival rates. In the present study, we identified various treatment modalities and their potential covariates. We also sought insight into the trends in survival for PCC during 2007-2015 in Ontario, after adjustments for cancer stage, treatments, and patient-specific factors.

\section{METHODS}

\section{Data Source}

This retrospective population-based cohort included patients 18 years of age and older diagnosed with primary invasive PCC (Statistical Classification of Diseases and Related Health Problems, revision 10 codes C25.x) from January 2007 to December 2015 whose disease stage was known. Neuroendocrine tumours (C25.4) constituted less than $0.01 \%$ of the identified population. Relevant patientbased covariates such as sex, age, date of diagnosis, survival, tumour characteristics, and treatment modalities were obtained from multiple administrative datasets held at ICES. Those datasets included the Ontario Cancer Registry (diagnosis date, stage, tumour characteristics), Cancer Activity Level Reporting [chemotherapy (CTX), radiation therapy (RT)], Discharge Abstract Database (surgery, RT, CTX), National Ambulatory Care Reporting System (CTX, RT), Ontario Health Insurance Plan claims (CTX, RT), sameday surgery datasets (CTX, RT), and the Registered Persons Database (age, sex, date of death). Those datasets were linked using unique encoded identifiers, and the data were analyzed at ICES.

Patients identified with in situ or noninvasive tumours based on the International Classification of Diseases for Oncology (2nd or 3rd edition) were not included in the analysis. Also, patients diagnosed solely based on a death certificate or autopsy were excluded.

Treatment modalities were investigated based on codes from the Canadian Classification of Health Interventions ${ }^{16}$ and the Canadian Classification of Diagnostic, Therapeutic, and Surgical Procedures ${ }^{17}$. Treatment modalities were classified as follows:

No treatment, if no record of treatment for the patient could be found

RT only, for patients who received only RT

CTx only, for patients who received only CTX

Chemoradiation (CRT), for patients who received both RT and CTX, but no surgical resection

Surgical resection, divided into

- distal pancreatectomy (DP) only, when no record of RT or CTX was found;

- DP plus RT or CTX;

- Whipple only, when no record of RT or CTx was found; and

- Whipple plus RT or CTx

To determine treatment classification, certain treatment groups were combined (that is, DP with RT, DP with CTX, and DP with CRT) if their Kaplan-Meier survival curves, adjusted for age and stage of disease, were similar. A treatment modality was included in the analysis only if it was received within 1 year after diagnosis.

Staging data have been made available in Ontario since 2007. Ontario Health (Cancer Care Ontario) uses a best-stage grouping approach based on pathology TNM when available and clinical TNM otherwise ${ }^{18}$. Stage is based on the criteria of the American Joint Committee on Cancer or the Collaborative Stage initiative. If a case had more than one valid stage value, the "best stage" was derived based on a pre-specified algorithm; otherwise, the stage group provided by the treating regional cancer centre, if available, was used ${ }^{19}$.

\section{Statistical Analysis}

Descriptive measures are reported based on treatment modality, demographics, and tumour characteristics. A logistic regression analysis was used to evaluate associations between demographics (sex and age groups), stage, or year of diagnosis and treatment modality. In the logistic regression model, the treatment modalities were regrouped as surgery compared with no surgery (reference group), and the effect of each variable is reported as an odds ratio (OR) with its $95 \%$ confidence interval (CI).

Next, we estimated the survival rate for patients diagnosed with PCC during 2007-2015, with follow-up to the end of 2016. We used a flexible parametric (Royston-Parmar) model to estimate the trends in survival rate for treatment modality during the study period ${ }^{20,21}$. The Royston-Parmar model uses restricted cubic splines on the log cumulative hazard scale to provide smooth estimates of survival. That approach has several advantages over the more standard survival models. It adopts a piecewise approach that is more flexible compared with the other traditional methods in mimicking the actual trends in mortality (hazard rate) and survival pattern ${ }^{22}$. We fitted a model by incorporating age group, sex, year of diagnosis, stage of tumour, treatment modality, and the interaction term between each pair of variables into a multiple statistical model using a forward approach. The likelihood ratio test was used to compare various models to reach to a final model. Then, based on the final model, trends in 1-, and 2-, and 5-year survival rates were estimated for each treatment modality while adjusting for the other covariates in the model. Each variable or interaction term was included in the final model if it was statistically significant ( $p \leq 0.05$ ).

The flexible parametric model was fitted using the freely available stpm 2 program written for the Stata software application. All statistical analyses were conducted using Stata (version MP 15.1: StataCorp LP, College Station, TX, U.S.A.).

\section{RESULTS}

The study included 6437 patients with primary PCC $(52.7 \%$ being men). Mean age at diagnosis was $66.4 \pm 11.4$ years. A slight increase in the number of patients diagnosed annually was observed, to 739 in 2015 from 655 in 2007.

Table I presents the distributions of age, stage of disease, and year of diagnosis based on treatment modality. In the study cohort, 1591 patients $(24.7 \%)$ received no treatment; 2084 (32.4\%) received CTx only; and 1571 (24.4\%) received 
TABLE I Demographics and tumour characteristics at diagnosis for patients with pancreatic cancer, based on treatment modality

\begin{tabular}{|c|c|c|c|c|c|c|c|c|c|}
\hline \multirow[t]{2}{*}{ Variable } & \multicolumn{9}{|c|}{ Modality [n (\%)] } \\
\hline & None & RT only & CTx only & CRT & DP only & $\begin{array}{l}\text { DP plus } \\
\text { RT or CTx }\end{array}$ & $\begin{array}{c}\text { Whipple } \\
\text { only }\end{array}$ & $\begin{array}{l}\text { Whipple plus } \\
\text { RT or CTx }\end{array}$ & TOTAL \\
\hline \multicolumn{10}{|l|}{ Age group } \\
\hline$<60$ Years & $265(15.3)$ & $58(3.4)$ & $614(35.5)$ & $286(16.5)$ & 79 (4.6) & $78(4.5)$ & $40(2.3)$ & $311(18.0)$ & 1731 \\
\hline 60-69 Years & 419 (20.9) & $65(3.2)$ & $699(34.9)$ & $302(15.1)$ & $44(2.2)$ & $81(4.0)$ & $33(1.7)$ & $362(18.1)$ & 2005 \\
\hline 70-79 Years & $488(26.0)$ & $98(5.2)$ & $596(31.7)$ & $246(13.1)$ & $28(1.5)$ & $67(3.6)$ & $68(3.6)$ & $289(15.4)$ & 1880 \\
\hline$\geq 80$ Years & $419(51.0)$ & $88(10.7)$ & $175(21.3)$ & $48(5.9)$ & $10(1.2)$ & $17(2.1)$ & $16(2.0)$ & $48(5.9)$ & 821 \\
\hline \multicolumn{10}{|l|}{ Sex } \\
\hline Men & $817(24.1)$ & $144(4.2)$ & $1143(33.7)$ & $474(14.0)$ & $67(2.0)$ & $118(3.5)$ & $82(2.4)$ & $550(16.2)$ & 3395 \\
\hline Women & $774(25.4)$ & $165(5.4)$ & $941(30.9)$ & $408(13.4)$ & $94(3.1)$ & $125(4.1)$ & $75(2.5)$ & $460(15.1)$ & 3042 \\
\hline \multicolumn{10}{|l|}{ Stage } \\
\hline I & 169 (32.0) & $26(4.9)$ & $42(8.0)$ & $42(8.0)$ & $87(16.5)$ & $47(8.9)$ & $33(6.3)$ & $82(15.5)$ & 528 \\
\hline II & $108(7.9)$ & $30(2.2)$ & $96(7.0)$ & $93(6.8)$ & $48(3.5)$ & $139(10.1)$ & $93(6.8)$ & 767 (55.8) & 1374 \\
\hline III & $194(19.2)$ & $71(7.0)$ & $307(30.4)$ & $342(33.9)$ & \multicolumn{2}{|c|}{$12(1.1)^{\mathrm{a}}$} & $8(0.8)$ & $76(7.5)$ & 1010 \\
\hline IV & 1120 (31.8) & $182(5.2)$ & 1639 (46.5) & 405 (11.5) & \multicolumn{2}{|c|}{$71(2.0)^{\mathrm{a}}$} & $23(0.7)$ & $85(2.4)$ & 3525 \\
\hline \multicolumn{10}{|l|}{ Year } \\
\hline 2007-2009 & $507(24.5)$ & $76(3.7)$ & $661(32.0)$ & 315 (15.3) & $35(1.7)$ & $71(3.4)$ & $51(2.5)$ & 350 (16.9) & 2066 \\
\hline 2010-2012 & 545 (24.5) & $117(5.3)$ & 687 (30.9) & $340(15.3)$ & $59(2.7)$ & $95(4.3)$ & $45(2.0)$ & $334(15.0)$ & 2222 \\
\hline 2013-2015 & $539(25.1)$ & $116(5.4)$ & $736(34.3)$ & 227 (10.6) & $67(3.1)$ & 77 (3.6) & $61(2.8)$ & $326(15.2)$ & 2149 \\
\hline TOTAL & $1591(24.7)$ & 309 (4.8) & 2084 (32.4) & $882(13.7)$ & $161(2.5)$ & $243(3.8)$ & $157(2.4)$ & 1010 & 6437 \\
\hline
\end{tabular}

a Because of small frequencies, these cells were combined to preserve anonymity.

$\mathrm{RT}=$ radiation therapy; $\mathrm{CTx}=$ chemotherapy; $\mathrm{CRT}=$ chemoradiation; $\mathrm{DP}=$ distal pancreatectomy .

a surgical intervention. Except for the $5.3 \%$ decrease in the proportion of patients receiving CRT during the study period, no notable changes were observed in the proportions of patients receiving various treatment modalities. Specifically, no notable change was observed in the proportion of patients receiving adjuvant or neoadjuvant CTX with a surgical intervention.

Table II presents the distributions of age, sex, treatment, and year of diagnosis based on stage. Overall, $54.7 \%$ of patients were diagnosed at stage IV and $8.2 \%$ at stage I. Only $13.1 \%(n=461)$ of patients with stage IV disease were 80 years of age and older, but those patients constituted $56.2 \%$ of that age group. When all patients diagnosed with PCC during a given time period were compared (Table II), we observed a slight increase in stage I disease and a decrease in stage III disease from 2007 to 2015.

Of the patients who received no treatment $(n=1591)$, $57.0 \%$ were 70 years of age or older and $16.7 \%$ were less than 60 years of age (Table II). Moreover, of all patients who received no treatment, $70.4 \%$ had stage IV disease, and $10.6 \%(n=169)$ had stage I disease. Further analysis showed that, of the patients with stage I disease who received no treatment, $88(52.1 \%)$ were 70 years of age or older, and 100 (59.2\%) were women.

Treatment modality also varied with age and cancer stage. Of the patients 80 or more years of age, $51.0 \%$ ( $n=$
419) received no treatment; of those less than 60 years of age, $15.3 \%$ received no treatment (Table I). However, based on further analysis, $64.2 \%$ of those 80 or more years of age who received no treatment had stage IV disease (data not shown). In addition, of all the patients 80 or more years of age who received no treatment, 99 had stage I or II disease, with $73 \%$ of those being women. Also, over time, we observed a slight increase in the proportion of patients 80 or more years of age who received no treatment: to $14 \%$ in 2015-2016 from 8\% in 2007-2008.

Surgical intervention was provided to $76.2 \%$ of patients with stage II disease, with or without RT or CTx (Table I). In contrast, $52.8 \%$ of patients with stage I disease $(n=279)$ received no surgical treatment. Upon further analysis, $35.5 \%$ of the patients with stage I disease who received no treatment $(n=169)$ were 80 or more years of age, and $22.5 \%$ were 70-79 years or age. From 2007 to 2015, no proportionate change was observed in patients with stage I disease who received no treatment.

\section{Factors Related to Surgical Resection}

Table III presents the factors associated with receipt of surgical resection in patients diagnosed with PCC. The likelihood of surgical resection receipt were not significantly different between men and women after adjustment for age, diagnosis year, and disease stage (OR: $0.94 ; 95 \%$ CI: 0.8 to 1.10 ). 
TABLE II Demographics and tumour characteristics at diagnosis for patients with pancreatic cancer, based on stage of disease

\begin{tabular}{|c|c|c|c|c|c|}
\hline \multirow[t]{2}{*}{ Variable } & \multicolumn{5}{|c|}{ Stage } \\
\hline & I & II & III & IV & TOTAL \\
\hline \multicolumn{6}{|l|}{ Age group } \\
\hline$<60$ Years & $163(30.9)$ & $369(26.9)$ & 238 (23.6) & $961(27.3)$ & $1731(26.9)$ \\
\hline 60-69 Years & $134(25.4)$ & $447(32.5)$ & 341 (33.8) & $1083(30.7)$ & $2005(31.2)$ \\
\hline 70-79 Years & $122(23.1)$ & $428(31.2)$ & $310(30.7)$ & $1020(28.9)$ & $1880(29.2)$ \\
\hline$\geq 80$ Years & 109 (20.6) & $130(9.5)$ & $121(12.0)$ & $461(13.1)$ & $821(12.8)$ \\
\hline \multicolumn{6}{|l|}{ Sex } \\
\hline Men & $242(45.8)$ & 699 (50.9) & $504(49.9)$ & $1950(55.3)$ & $3395(52.7)$ \\
\hline Women & $286(54.2)$ & $675(49.1)$ & $506(50.1)$ & $1575(44.7)$ & $3042(47.3)$ \\
\hline \multicolumn{6}{|l|}{ Treatment modality } \\
\hline None & $169(32.0)$ & $108(7.9)$ & $194(19.2)$ & $1120(31.8)$ & $1591(24.7)$ \\
\hline RT only & $26(4.9)$ & $30(2.2)$ & $71(7.0)$ & $182(5.2)$ & 309 (4.8) \\
\hline CTx only & $42(8.0)$ & $96(7.0)$ & 307 (30.4) & $1639(46.5)$ & $2084(32.4)$ \\
\hline CRT & $42(8.0)$ & $93(6.8)$ & $342(33.9)$ & $405(11.5)$ & $882(13.7)$ \\
\hline DP only & $87(16.5)$ & $48(3.5)$ & \multicolumn{2}{|c|}{$26(0.6)^{\mathrm{a}}$} & $161(2.5)$ \\
\hline DP plus RT or CTx & $47(8.9)$ & 139 (10.1) & \multicolumn{2}{|c|}{$57(1.3)^{\mathrm{a}}$} & $243(3.8)$ \\
\hline Whipple only & $33(6.3)$ & $93(6.8)$ & $8(0.8)$ & $23(0.7)$ & $157(2.4)$ \\
\hline Whipple plus RT or CTx & $82(15.5)$ & $767(55.8)$ & $76(7.5)$ & $85(2.4)$ & $1010(15.7)$ \\
\hline \multicolumn{6}{|l|}{ Year } \\
\hline 2007-2009 & $142(26.9)$ & $450(32.8)$ & $378(37.4)$ & $1096(31.1)$ & $2066(32.1)$ \\
\hline 2010-2012 & 179 (33.9) & $466(33.9)$ & $353(35.0)$ & $1224(34.7)$ & $2222(34.5)$ \\
\hline 2013-2015 & $207(39.2)$ & $458(33.3)$ & $279(27.6)$ & $1205(34.2)$ & $2149(33.4)$ \\
\hline TOTAL & 528 & 1374 & 1010 & 3525 & 6437 \\
\hline
\end{tabular}

a Because of small frequencies, these cells were combined to preserve anonymity.

$\mathrm{RT}=$ radiation therapy; $\mathrm{CTx}=$ chemotherapy; $\mathrm{CRT}=$ chemoradiation; $\mathrm{DP}=$ distal pancreatectomy

TABLE III Predictors of surgical resection in patients diagnosed with pancreatic cancer

\begin{tabular}{|c|c|c|}
\hline Variable & OR & $95 \% \mathrm{Cl}$ \\
\hline \multicolumn{3}{|l|}{ Sex } \\
\hline Men & \multicolumn{2}{|c|}{ Referent } \\
\hline Women & 0.94 & 0.80 to 1.10 \\
\hline \multicolumn{3}{|l|}{ Age group } \\
\hline$<60$ Years & \multicolumn{2}{|c|}{ Referent } \\
\hline $60-69$ Years & 0.75 & 0.61 to $0.92^{\mathrm{a}}$ \\
\hline 70-79 Years & 0.60 & 0.49 to $0.74^{b}$ \\
\hline$\geq 80$ Years & 0.17 & 0.12 to $0.23^{b}$ \\
\hline Year of diagnosis & 0.99 & 0.96 to 1.03 \\
\hline \multicolumn{3}{|l|}{ Stage } \\
\hline I & \multicolumn{2}{|c|}{ Referent } \\
\hline II & 3.4 & 2.72 to $4.24^{b}$ \\
\hline III & 0.10 & 0.08 to $0.13^{b}$ \\
\hline IV & 0.05 & 0.04 to $0.06^{b}$ \\
\hline
\end{tabular}

a $p<0.05$.

b $p<0.001$.

$\mathrm{OR}=$ odds ratio; $\mathrm{Cl}=$ confidence interval.
The adjusted odds of surgical resection receipt did not change over time (OR: 0.99; 95\% CI: 0.96 to 1.03). Compared with patients less than 60 years of age, those $60-69$ years of age (OR: $0.75 ; 95 \%$ CI: 0.61 to 0.92 ), $70-79$ years of age (OR: $0.60 ; 95 \%$ CI: 0.49 to 0.74 ), and 80 or more years of age (OR: 0.17 ; $95 \%$ CI: 0.12 to 0.23 ) had a significantly lower chance of receiving surgical resection after adjustment for stage, sex, and year of diagnosis.

After adjustment for sex, age group, and year of diagnosis, significantly more patients with stage II disease than with stage I disease received a surgical intervention (OR: 3.4; 95\% CI: 2.72 to 4.24 ), and significantly fewer of those with stage III (OR: $0.10 ; 95 \%$ CI: 0.08 to 0.13 ) and stage IV disease (OR: $0.05 ; 95 \%$ CI: 0.04 to 0.06 ) than with stage I disease received a surgical intervention.

\section{Trends in Survival Based on Treatment Modality}

Overall, 1-year survival increased to $42 \%$ from $33 \%$; 2 -year survival increased to $26 \%$ from $15 \%$; and 5 -year survival increased to $15 \%$ from $7 \%$ (Figure 1 ).

Figure 2 shows trends in survival based on treatment modality, after adjustment for age, sex, and stage. No changes in 1-, 2-, and 5-year survival were observed for the no-treatment and RT-only groups. Of all the treatment groups, the group that underwent DP ONLY had the 


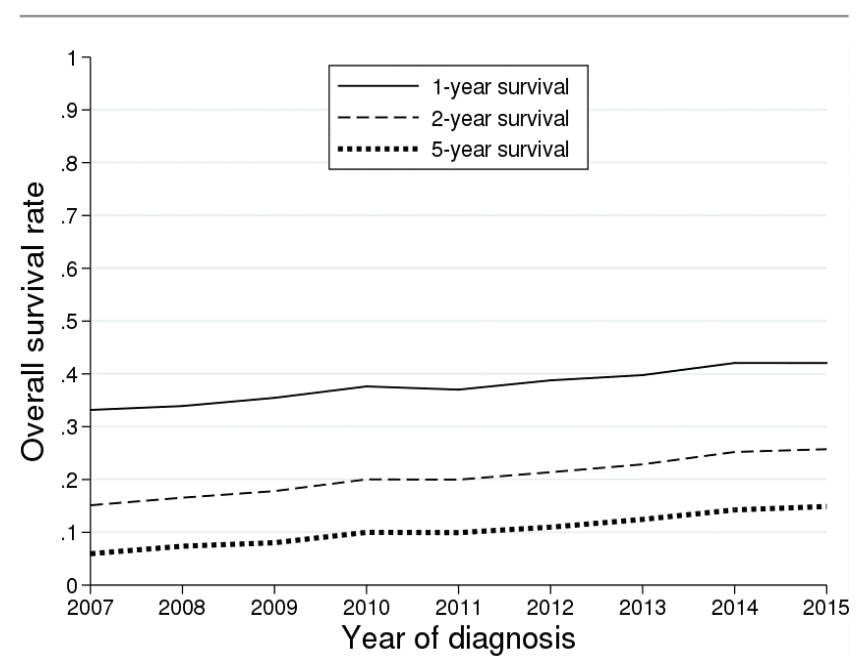

FIGURE 1 Trends in overall 1-, 2-, and 5-year survival rates adjusted for age, sex, disease stage, and treatment modality from 2007 to 2015.
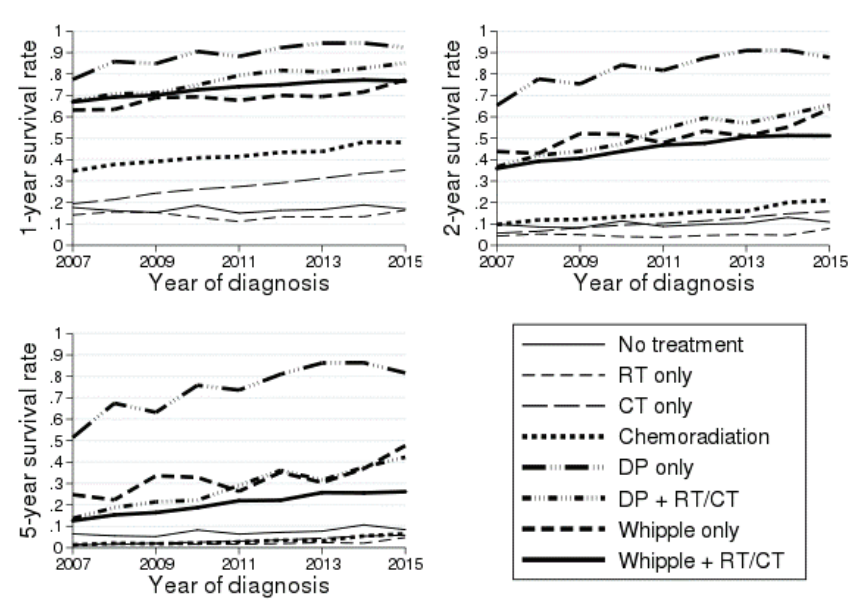

FIGURE 2 Adjusted trends in 1-year, 2-year, and 5-year survival rates from 2007 to 2015, based on treatment modality. RT = radiation therapy; $\mathrm{CT}=$ chemotherapy; $\mathrm{DP}=$ distal pancreatectomy.

highest 1-, 2-, and 5-year survival. For the DP-only group, 1 -year survival increased by 14 percentage points (to $92 \%$ from $78 \%$ ); 2 -year survival, by 24 percentage points (to $89 \%$ from $65 \%$ ); and 5 -year survival, by 29 percentage points (to $81 \%$ from $52 \%$ ). In contrast, the survival rates for DP plus RT or CTX were lower than those for DP only, with 1-year survival increasing by 16 percentage points (to $84 \%$ from $68 \%$ ); 2 -year survival, by 28 percentage points (to $65 \%$ from $37 \%$ ); and 5 -year survival by 29 percentage points (from $12 \%$ to $41 \%$ ).

Similarly, the survival rate for Whipple-only treatment was higher than that for Whipple plus RT or CTx. For Whipple only, 1-year survival increased by 16 percentage points (to $78 \%$ from $62 \%$ ); 2 -year survival, by 16 percentage points (to $60 \%$ from $44 \%$ ); and 5 -year survival, by 24 percentage points (to $49 \%$ from $25 \%$ ). In contrast, 1-year survival for Whipple plus RT or CTx increased by 9 percentage points (to $77 \%$ from $68 \%$ ); 2 -year survival, by 14 percentage points

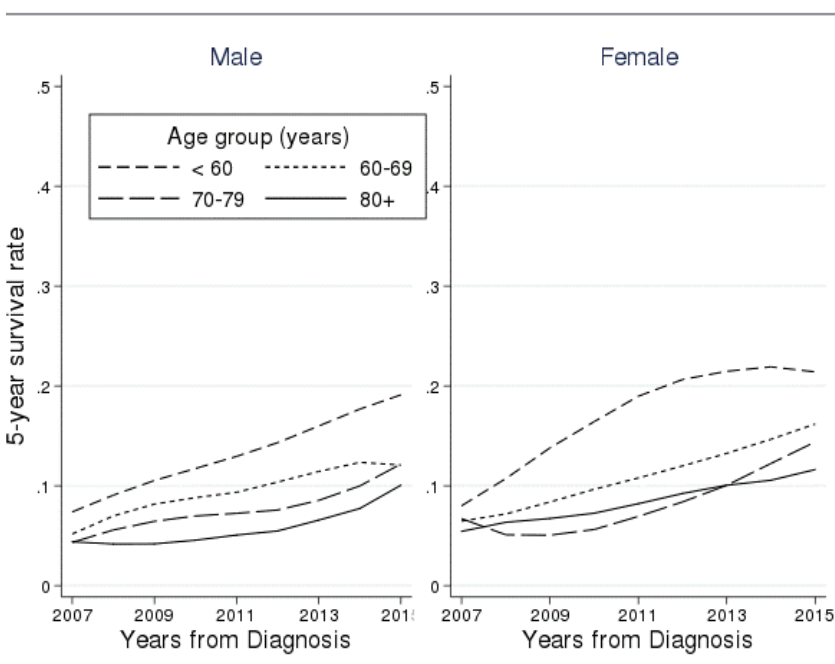

FIGURE 3 Adjusted trends in 1-year, 2-year, and 5-year survival rates from 2007 to 2015, based on age group and sex.

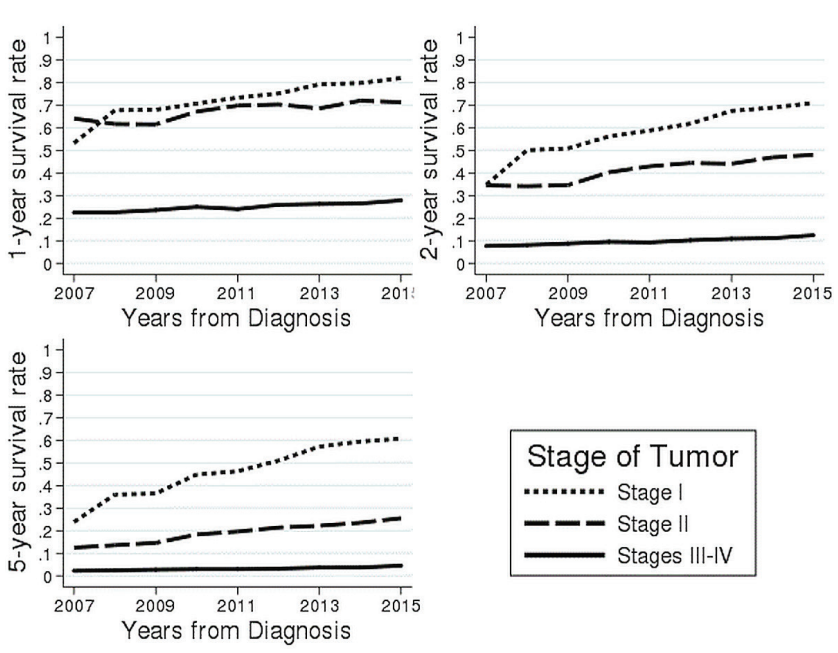

FIGURE 4 Adjusted trends in 1-year, 2-year, and 5-year survival rates from 2007 to 2015, based on stage of disease.

(to $50 \%$ from $36 \%$ ); and 5 -year survival, by 14 percentage points (to $26 \%$ from $12 \%$ ).

The 1-year survival rate for CTX increased only by 15 percentage points (to $35 \%$ from $20 \%$ ); 2 -year survival, by 4 percentage points (to $9 \%$ from $5 \%$ ); and 5 -year survival, by 3 percentage points (to $4 \%$ from $1 \%$ ). The survival rate for the CRT group was higher than that for the CTX-only group. The 1-year survival rate for CRT increased to $49 \%$ from $35 \%$; the 2 -year rate, to $21 \%$ from $10 \%$; and the 5 -year rate, to $7 \%$ from $1 \%$.

\section{Trends in Survival Based on Sex and Age Group}

Figure 3 shows that 5 -year survival rates increased for all age groups and for men and women. The highest increases were observed in men less than 60 years of age (11 percentage points, to $19 \%$ from $8 \%$ ) and in women less than 60 years of age (14 percentage points, to $22 \%$ from $8 \%$ ). 


\section{Trends in Survival Based on Stage of Disease}

Figure 4 shows trends in survival based on tumour stage and adjusted for sex, age, treatment modality, and year of diagnosis. For stage I disease, 1-year survival increased by 30 percentage points (to $81 \%$ from $51 \%$ ); 2 -year survival, by 36 percentage points (to $71 \%$ from $35 \%$ ); and 5 -year survival, by 39 percentage points (to $61 \%$ from $22 \%$ ). For stage II disease, 1-year survival increased by 8 percentage points (to $71 \%$ from $63 \%$ ); 2 -year survival, by 14 percentage points (to $49 \%$ from $35 \%$ ); and 5 -year survival, also by 14 percentage points (to 25\% from 11\%). Lastly, for stages III and IV disease, 1-year survival increased by 8 percentage points (to $29 \%$ from $21 \%$ ); 2 -year survival, by 3 percentage points (to $22 \%$ from $19 \%$ ); and 5 -year survival, by 3 percentage points (to $5 \%$ from $1 \%)$.

\section{DISCUSSION}

This population-based retrospective study provides an overview of positive trends in the survival of patients with PCC in Ontario from 2007 to 2015 . Our study found no noticeable improvement in survival for patients with stage III and IV tumours, who constitute the highest proportion of patients with PCC. However, the most improvement in survival was observed for surgical modalities and for patients with stage I disease. Specifically, 2-year and 5-year survival rates increased by 25-30 percentage points in the DP only, DP plus RT or CTX, and Whipple-only groups. The 2 - and 5 -year survival rates improved by $10-15$ percentage points for the Whipple plus RT or CTx group. Similarly, the 1-, 2-, and 5-year survival rates increased by 30-39 percentage points for stage I disease. The 1-year survival for the CTXonly and CRT groups increased by 14 percentage points. Our study demonstrates that survival has increased and varies by treatment modality, even after adjustment for patient sex and age, year of diagnosis, and stage of disease. Our observations imply that the improvements in survival based on treatment modality in our study cannot be attributed to stage migration or changes in patient-specific factors over time.

The results of our study pertaining to improvements in survival for PCC are consistent with those previously published in population-based studies-albeit with limitations in the prior literature with respect to adjustment for stage or treatment-specific factors, or both. In a U.S. Surveillance, Epidemiology, and End Results database analysis reporting on survival rates from 1981-1990 to 2001-2010 in the United States, 1-year relative survival increased to $28.2 \%$ from $17 \%^{15}$. In contrast, the change in 5 -year relative survival was minimal (to $6.9 \%$ from $3.1 \%$ ). Another international comparison of survival rates between 2003 and 2014 in the United States, the Netherlands, Norway, Belgium, and Slovenia showed that the unadjusted 1-year survival rate for European countries ranged from $19 \%$ to $34 \%{ }^{23}$. In contrast, the unadjusted 5-year survival rate ranged from $4 \%$ to $11 \%$ for European countries and from $7 \%$ to $10 \%$ for the United States $^{9,23,24}$. In our study, overall 1-year survival increased to $42 \%$ from $33 \%$; 2 -year survival increased to $26 \%$ from $16 \%$; and 5 -year survival increased to $15 \%$ from $7 \%$ (Figure 1), showing considerably higher survival in Ontario. However, a direct comparison of survival rates between the foregoing studies is difficult, given the difference in the fiscal years compared. In addition, our study presents survival rates adjusted for patient age, sex, treatment, and stage, which allows for adjustment of shift in stage, age of diagnosis, or treatment modalities over time.

It is well established that survival in PCC is higher for resectable tumours. The highest 5 -year survival-81\% in 2015-was observed for the DP-only group, and the lowest-26\% - was observed for the Whipple plus RT or CTX group. In addition, when patients underwent DP together with CTX or RT, or both, the adjusted survival was similar to that for the patients who underwent a Whippleonly procedure. Our sub-analysis shows that $54 \%$ of all patients who underwent DP-only had stage I disease, and $55 \%$ of those who underwent DP plus CTx or RT had stage II disease. However, the survival difference persisted even after adjusting for age, sex, and stage, implying that other factors not accounted for in the model are affecting survival (for example, margin status or neoadjuvant therapy, among others). In addition, survival rates after resection were slightly higher in our study than have previously been reported. An international multicentre cohort study from 5 tertiary centres in Europe and the United States (2000-2015) evaluated 5-year survival for patients who underwent pancreaticoduodenectomy (Whipple procedure) for nonmetastatic pancreatic ductal adenocarcinoma ${ }^{25}$. For patients who received no neoadjuvant treatment, that study reported survival rates of $39 \%$ for stage IA, $34 \%$ for stage IB, $27 \%$ for stage IIA, $21 \%$ for stage IIB, and $10 \%$ for stage III. The 5 -year survival rates in our study, after adjusting for stage, age, and neoadjuvant or adjuvant treatments, were $25 \%$ - 49\% for Whipple-only (2007-2015) and $12 \%-26 \%$ for Whipple plus RT or CTX. Although a direct comparison is difficult given the differences in statistical analyses, time period, and tumour selection criteria, those rates do highlight a slightly higher adjusted 5-year survival rate for Whipple-only surgeries in Ontario.

A review of the literature suggests that the existing studies reported on survival rates for PCC either as unadjusted rates or as stratified by stage, age, or specific treatment modalities ${ }^{23,25,26}$. Although presentation of stratified survival can assist in patient counselling, it does not allow for comparison of overall survival rates over time. Our analyses therefore focused on providing 1-, 2-, and 5-year survival rates adjusted for patient-, tumour-, and treatmentspecific factors. The results of our analysis highlight the important finding that, even after adjusting for the effect of any change in patient age, disease stage, or treatment offered to the patient, a considerable improvement in survival remains - an improvement potentially related to factors not available for analysis in the present study. Those factors include, but are not limited to, improvements in the quality of surgical treatment, reduction in surgical complication rates, improvements in postoperative mortality, or reduced margin involvement after a resection. Also, better combinations of existing chemotherapeutic agents, better imaging techniques leading to earlier detection and management, advancement in surgical techniques for resectable tumours, and introduction of neoadjuvant therapy leading to feasibility of surgery in patients with borderline resectable or locally resectable disease could 
have led to improved survival for patients receiving surgical modalities ${ }^{5,10,27}$. For nonresectable tumours, the improvement in survival seen in the CTX-only group, as well as in the CRT group, could be attributable to better patient selection-that is, CTx was provided to patients with an acceptable performance status, thus improving survival. In the absence of any new CTX agents or biomarkers for the early detection of PCC, the explanation for the increase in adjusted survival likely lies in better patient selection and improvements in the quality of care delivered.

In a universally funded health care system, it is imperative to ensure equitable delivery of high-quality care to all eligible patients. Our study found that a large proportion of patients 80 years of age and older received no treatment. Although that finding could be explained by the fact that most (56\%) had been diagnosed with stage IV disease, it does not explain the patients with stage I or II disease in that age group who received no treatment. Because we lacked access to performance status, comorbidities, and patient preferences for treatment, we cannot speculate whether the best available management options were provided to those patients. There was also a slight increase in the proportion of patients 80 years of age and older who received no treatment: $14 \%$ in $2015-2016$ compared with $8 \%$ in 2007-2008. Our study found improvements in survival for patients who received CTX. Further research is needed to determine the appropriateness of treatment for older adults with PCC to ensure equitable access to the best available treatment modalities.

The strength of our study is the availability of cancer staging and treatment-specific data for a large number of patients. Only a few studies have reported survival trends in patients with PCC, and to our knowledge, the present study is the first of its kind in Canada. The limitations of our analysis include a lack of information about the type of CTX administered; our inability to differentiate between neoadjuvant and adjuvant chemotherapies; and inadequate information to determine the nature of RT-that is, whether it was administered for palliative care purposes at any point during disease management. Incomplete submission of cancer staging data for all patients diagnosed with PCC between 2007 and 2015 in Ontario prevented use of the full cohort in the analyses. Also, we were not able to capture and use important information such as comorbidity and patient preferences in the analyses. Furthermore, the study is susceptible to coding and recording error, a limitation inherent to most studies based on administrative datasets. Finally, our study was conducted within a single-payer health care system and its findings might not be generalizable to private health care systems.

\section{CONCLUSIONS}

Survival in PCC is highly dependent on treatment modality and cancer stage at diagnosis. Positive trends in short-term survival for early-stage disease and surgical modalities are evident at the population level. Further research is needed to identify factors associated with observed improvements in survival over time that could not be explained by patient age, sex, stage, treatment, and year of diagnosis. Lastly, development and implementation of better CTX agents is a continuing need to improve survival for the large group of patients diagnosed with stages III and IV disease.

\section{ACKNOWLEDGMENTS}

This study was supported by ICES, which is funded by an annual grant from the Ontario Ministry of Health (мон). The Ontario Institute for Cancer Research paid for data linkage between the various databases. The opinions, results, and conclusions reported in this paper are those of the authors and are independent from the funding sources. No endorsement by ICES or the Ontario мон is intended or should be inferred. Parts of this material are based on data or information compiled and provided by the Canadian Institutes of Health Research (CIHI); however, the analyses, conclusions, opinions, and statements expressed in the material are those of the authors, and not necessarily those of CIHI. Also, parts of this material are based on data and information provided by Ontario Health (Cancer Care Ontario) [оH(CCO)]. The opinions, results, view, and conclusions reported in this paper are those of the authors and do not necessarily reflect those of $\mathrm{OH}(\mathrm{CCO})$. No endorsement by OH(CCO) is intended or should be inferred. We are indebted to ICES staff at McMaster-specifically, to Richard Perez and Joshua Cerasuolo-for their invaluable help and support.

\section{CONFLICT OF INTEREST DISCLOSURES}

We have read and understood Current Oncology's policy on disclosing conflicts of interest, and we declare that we have none.

\section{AUTHOR AFFILIATIONS}

*School of Medicine, University of Toronto, Toronto, ON; ${ }^{\dagger}$ Departments of Surgery, ${ }^{\ddagger}$ Health Research Methods, Evidence, and Impact, and ${ }^{\S}$ Oncology, McMaster University, Hamilton, ON; "School of Nursing, McMaster University, Hamilton, ON.

\section{REFERENCES}

1. Ellison L.Age-Specific Patterns in the Incidence of, and Survival from, Pancreatic Cancer in Canada. Ottawa, ON: Statistics Canada; 2017.

2. Collier R. Half of Canadians can expect cancer diagnosis during lifetime. CMAJ 2017;189:E920.

3. American Cancer Society (ACS). Cancer Facts and Figures 2017. Atlanta, GA: ACs;2017.

4. Padbury RT. Survival from pancreatic cancer: it's not just about the surgical mortality. Med J Aust 2012;196:491-2.

5. Paulson AS, Tran Cao HS, Tempero MA, Lowy AM. Therapeutic advances in pancreatic cancer. Gastroenterology 2013; 144:1316-26.

6. Porta M, Fabregat X, Malats N, et al. Exocrine pancreatic cancer: symptoms at presentation and their relation to tumour site and stage. Clin Transl Oncol 2005;7:189-97.

7. Sauer BG, Shami VM. Survival in pancreatic cancer: the best is yet to come. Gastrointest Endosc 2010;72:84-5.

8. Hartwig W, Werner J, Jager D, Debus J, Buchler MW. Improvement of surgical results for pancreatic cancer. Lancet Oncol 2013;14:e476-85.

9. Sirri E, Castro FA, Kieschke J, et al. Recent trends in survival of patients with pancreatic cancer in Germany and the United States. Pancreas 2016;45:908-14.

10. Ansari D, Gustafsson A, Andersson R. Update on the management of pancreatic cancer: surgery is not enough. World J Gastroenterol 2015;21:3157-65.

11. Akhtar-Danesh GG, Finley C, Akhtar-Danesh N. Long-term trends in the incidence and relative survival of pancreatic cancer in Canada: a population-based study. Pancreatology 2016;16:259-65. 
12. Luke C, Price T, Karapetis C, Singhal N, Roder D. Pancreatic cancer epidemiology and survival in an Australian population. Asian Pac J Cancer Prev 2009;10:369-74.

13. Quaresma M, Coleman MP, Rachet B. 40-Year trends in an index of survival for all cancers combined and survival adjusted for age and sex for each cancer in England and Wales, 1971-2011: a population-based study. Lancet 2015;385: 1206-18.

14. Soreide K, Aagnes B, Moller B, Westgaard A, Bray F. Epidemiology of pancreatic cancer in Norway: trends in incidence, basis of diagnosis and survival 1965-2007. Scand J Gastroenterol 2010;45:82-92.

15. Sun H, Ma H, Hong G, Sun H, Wang J. Survival improvement in patients with pancreatic cancer by decade: a period analysis of the SEER database, 1981-2010. Sci Rep 2014;4:6747.

16. Canadian Institute for Health Information (CIHI). Canadian Classification of Health Interventions. Ottawa, ON: CIHI; 2015.

17. Statistics Canada. Canadian Classification of Diagnostic, Therapeutic, and Surgical Procedures. Ottawa, ON: Statistics Canada;1986.

18. Booth CM, Li G, Zhang-Salomons J, Mackillop WJ. The impact of socioeconomic status on stage of cancer at diagnosis and survival: a population-based study in Ontario, Canada. Cancer 2010;116:4160-7.

19. Ginsburg OM, Fischer HD, Shah BR, et al. A population-based study of ethnicity and breast cancer stage at diagnosis in Ontario. Curr Oncol 2015;22:97-104.
20. Royston P, Lambert PC. Flexible Parametric Survival Analysis Using Stata: Beyond the Cox Model. College Station, TX: Stata Press; 2011.

21. Royston P, Parmar MK. Flexible parametric proportionalhazards and proportional-odds models for censored survival data, with application to prognostic modelling and estimation of treatment effects. Stat Med 2002;21:2175-97.

22. Rutherford MJ, Dickman PW, Lambert PC. Comparison of methods for calculating relative survival in population-based studies. Cancer Epidemiol 2012;36:16-21.

23. Huang L, Jansen L, Balavarca Y, et al. Stratified survival of resected and overall pancreatic cancer patients in Europe and the U.S.A. in the early twenty-first century: a large, international population-based study. BMCMed 2018;16:125.

24. Brenner H, Gondos A, Arndt V. Recent major progress in longterm cancer patient survival disclosed by modeled period analysis. J Clin Oncol 2007;25:3274-80.

25. van Roessel S, Kasumova GG, Verheij J, et al. International validation of the eighth edition of the American Joint Committee on Cancer (AJCC) TNM staging system in patients with resected pancreatic cancer. JAMA Surg 2018;153:e183617.

26. van der Geest LGM, van Eijck CHJ, Groot Koerkamp B, et al. Trends in treatment and survival of patients with nonresected, nonmetastatic pancreatic cancer: a population-based study. Cancer Med 2018;7:4943-51.

27. Mohammed S, Van Buren G 2nd, Fisher WE. Pancreatic cancer: advances in treatment. World J Gastroenterol 2014; 20:9354-60. 